\title{
Plasmonic metal-cored fibres
}

\author{
Behrad Gholipour ${ }^{1 *}$, Duc Minh Nguyen ${ }^{1,3^{*}}$, Paul Bastock ${ }^{2}$, Long Cui ${ }^{1}$, Venkatram Nalla ${ }^{1}$, Chris Craig $^{2}$, \\ Khouler Khan ${ }^{2}$, Daniel W. Hewak ${ }^{2}$, Nikolay I. Zheludev ${ }^{1,2}$ and Cesare Soci ${ }^{1 *}$ \\ ${ }^{1}$ Centre for Disruptive Photonic Technologies, Nanyang Technological University, Singapore, 637371 \\ ${ }^{2}$ Optoelectronics Research Centre, University of Southampton, Southampton SO17 1BJ, UK \\ ${ }^{3}$ CINTRA CNRS/NTU/Thales, UMI 3288, Research Techno Plaza, Singapore 637553 \\ email:b.gholipour@ntu.edu.sg,DMNguyen@ntu.edu.sg,csoci@ntu.edu.sg
}

\begin{abstract}
We study plasmonic metal-cored glass fibres where, upon pulsed laser excitation, the coupling between plasmonic and dielectric modes induces strong light collimation and suppression of nonlinear absorption, providing a novel platform for tunable fiberized sources and sensors.

OCIS codes: (060.2310) Fiber Optics; (250.5403) Plasmonics; (190.0190) Nonlinear Optics, (320.0320) Ultrafast Optics
\end{abstract}

Since the turn of the century, multimode fibres have evolved from all glass structures to a multi-material realm where hybrid fibers composed of conductors, semiconductors and insulator cores in conjunction with predominantly polymer clads have been demonstrated. This has given rise to a wide variety of novel applications including metamaterials, light sources, photodetectors, sensors as well as medical imaging devices. Such fibres may be realized with a range of functional materials including metallic, amorphous, crystalline, and semiconducting alloys $[1,2]$.
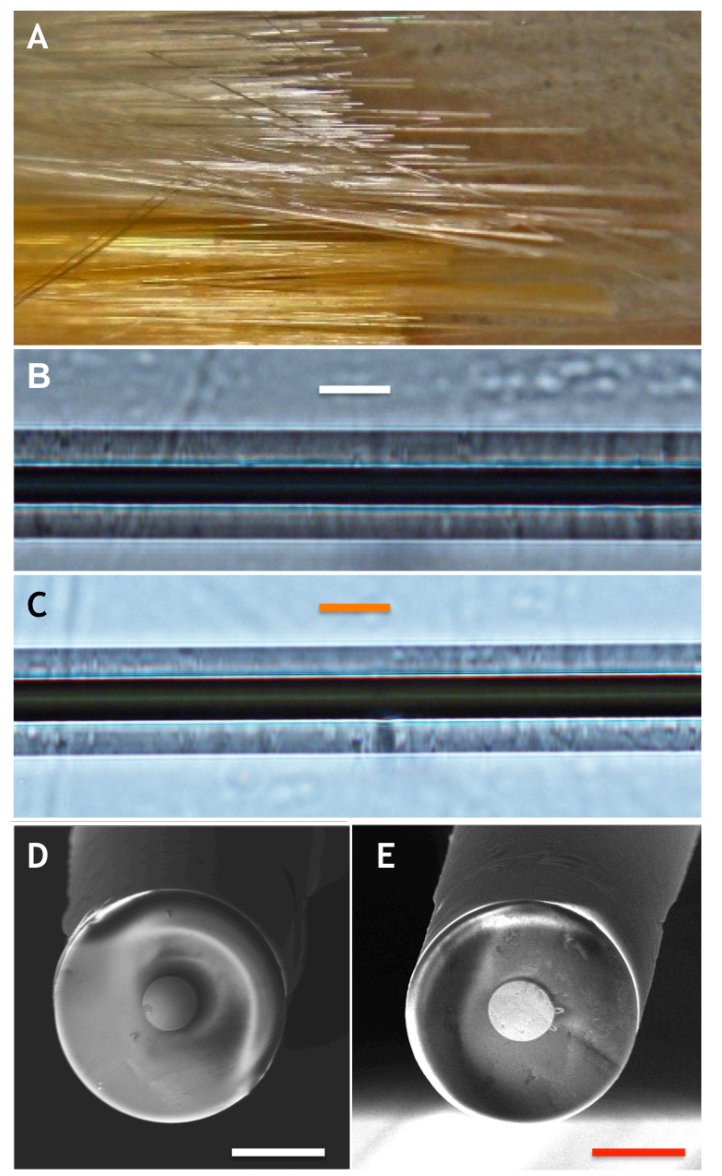

Fig. 1. Metal cored Optical fibers. A) Camera image of $\mathrm{Au}$ and Ag-cored fiber bundles. B, C) Side-view Tranmission optical microscope images of Ag and Au-cored fibers respectively. Scale bars indicate $10 \mu \mathrm{m}$. D, E) Scanning electron microscopy of typical fabricated Au-cored and Agcored silicate clad fiber, respectively.
Here we report our studies on multimaterial fibres with metallic core constituents, a novel platform for the study and utilisation of light-matter interaction under all-around, highly confined excitation of the functional cores over ultra-long macroscopic distances. We show that, upon injection of ultrashort laser pulses, the large fields localised in the plasmonic core strongly alter the overall mode profile and power response of the fibre due to the formation of a hybrid (plasmonic/dielectric) mode. As such, these hybrid fibre structures present a unique route to merge the fields of multimaterial fibres and fibre lasers with plasmonics, with potential applications in signal processing, sensing and sub-wavelength-scale optical imaging.

We fabricated multimode silicate glass optical fibres with $\mathrm{Au}$, $\mathrm{Ag}$ and $\mathrm{Sn}$ cores with radii down to $4 \mu \mathrm{m}$ and cladding outer radii down to $15 \mu \mathrm{m}$ by a Taylor-wire process [3] (Fig. 1). The metal-dielectric structure of such silicate fibres is expected to support propagation of both plasmonic and dielectric modes. To investigate the weight of plasmonic effects on the overall fibre response we compared fibres with "good" plasmonic metal cores, $\mathrm{Au}\left(\lambda_{\mathrm{P}}=540 \mathrm{~nm}\right)$ and $\mathrm{Ag}\left(\lambda_{\mathrm{P}}=380 \mathrm{~nm}\right)$, and with Sn core, which is known to be a "poor" plasmonic metal.

To investigate the interaction between the plasmonic and dielectric modes, short laser pulses (100 fs pulse width at $1 \mathrm{KHz}$ repetition rate) were injected into the fibres, and both the output beam profile and the input/output characteristics were collected across the visible spectral range (400 to $700 \mathrm{~nm}$ ) at different pump intensities.

Notably, a highly collimated output beam was observed in these experiments for a broad range of excitation intensities and wavelengths. Fig. 2a shows the comparison between the output beam intensity profiles collected in the far field, at increasing distances from the tip of a bare silicate fibre, the Au- and the Sncored silicate fibres. All fibres used in the experiment had the same outer radius of $15 \mu \mathrm{m}$. While the output beam of the bare 
fibre diverges so quickly that it becomes as large as the CCD sensor area at a distance of $5 \mathrm{~cm}$, in the case of the Au core the intensity profile of the output beam shows much greater collimation, with narrow focusing at a distance of 8 $\mathrm{cm}$. The corresponding output beam divergences are $\sim 10 \mathrm{mrad}$ and $70 \mathrm{mrad}$ for the Au-cored silicate fibre and the bare silicate fibre, respectively. We attribute the self-collimation properties of the Au-cored fibre to the selection of the lowest order modes induced by the high-intensity pulses. This is confirmed by comparison with the output from the Sn-cored fibre, which, due to poor plasmonic properties, shows much poorer collimation (divergence of $\sim 66$ $\mathrm{mrad})$.

Furthermore measurements of the power response function $\left(\mathrm{P}_{\text {out }}\right.$ vs $\left.\mathrm{P}_{\text {in }}\right)$ at various wavelengths from $400 \mathrm{~nm}$ to 700 $\mathrm{nm}$ for Au-cored fibre shown in Fig. $2 \mathrm{~b}$ show nonlinear absorption across different frequencies, with gain-like behaviour observed at $480 \mathrm{~nm}$ and $650 \mathrm{~nm}$, which correspond to the effective index matching position between plasmonic and dielectric modes propagating along the fibres. The effective gain is due to nonlinear effects resulting from the weak coupling between the plasmonic and dielectric modes.
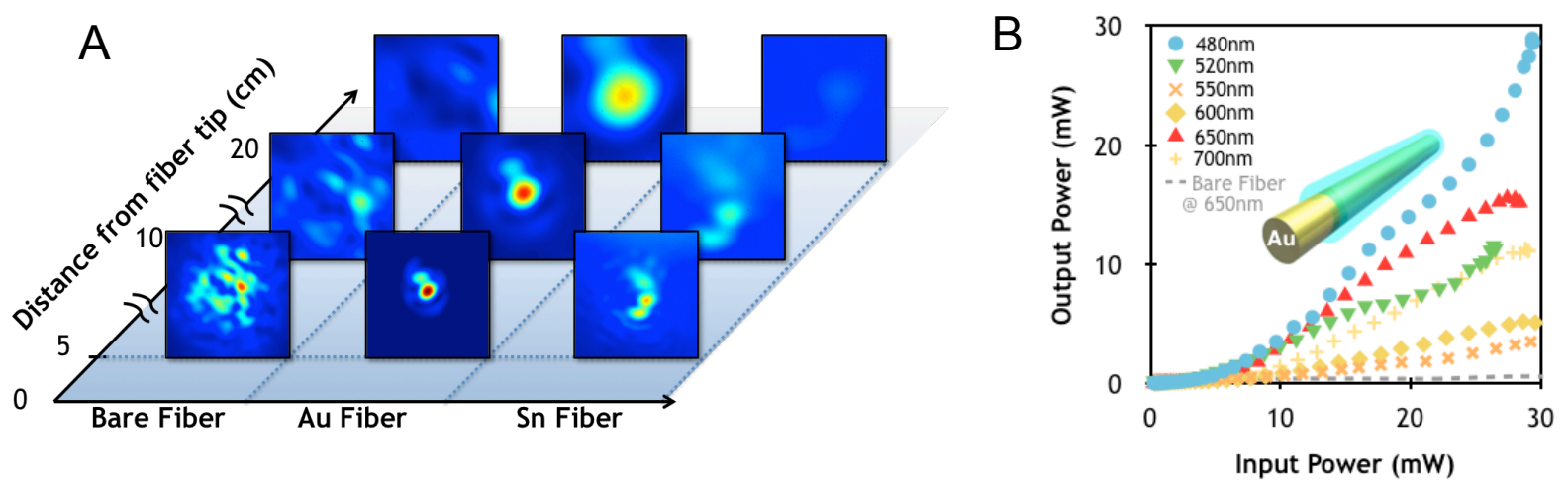

Fig. 2 Measured propagated modal profile and power response of output beam from fiber tip. (a) $2 D$ far field images at different $C C D$ camera positions with respect to the fiber tip, for three different cases: bare silicate fiber, plasmonic Au-cored fiber and Sn-cored. (b) Power response of Au-cored fiber at different wavelength in comparison with bare fiber at $650 \mathrm{~nm}$.

In conclusion, we fabricated a variety of metallic core/silicate glass clad fibres that exhibit a highly collimated output and nonlinear power response across a range of frequencies when pumped with intense laser pulses. Such hybrid fibre structures provide a perfect architecture for enhancing light-matter interaction by quasi-continuous excitation of the active cores. Strong energy confinement at the core/silicate interface induced by the excitation of surface plasmon polaritons may offer a new platform for the development of plasmonically enhanced fiberized sources and sensors.

\section{References}

[1] J. R. Sparks, R. He, N. Healy, M. Krishnamurthi, A. C. Peacock, P. J. A. Sazio, V. Gopalan, and J. V. Badding, Adv. Mater. 23, $1647-$ 1651 (2011).

[2] J. Ballato, T. Hawkins, P. Foy, R. Stolen, B. Kokuoz, M. Ellison, C. McMillen, J. Reppert, A. M. Rao, M. Daw, S. R. Sharma, R. Shori, O. Stafsudd, R. R. Rice, and D. R. Powers, Opt. Express 16, 18675 (2008).

[3] G. F. Taylor, Physical Review 23, 655-660 (1924). 